\title{
Calculation of clinical dose distributions in proton therapy from microdosimetry
}

Bertolet A. ${ }^{1,2}$, Cortés-Giraldo M.A. ${ }^{2}$, Souris K. ${ }^{3}$, Cohilis M. ${ }^{3}$, Carabe A. ${ }^{1}$

${ }^{1}$ Department of Radiation Oncology, Hospital of The University of Pennsylvania, Philadelphia, PA USA

${ }^{2}$ Department of Atomic, Molecular and Nuclear Physics, Universidad de Sevilla, Seville, Spain

${ }^{3}$ Université Catholique de Louvain, Center for Molecular Imaging and Experimental Radiotherapy, Louvain, Belgium.

Corresponding author: Alejandro Carabe-Fernandez. Department of Radiation Oncology, Hospital of The University of Pennsylvania, Philadelphia, 19104, PA, USA.

E-mail: a.carabe@uphs.upenn.edu; alexcarabe@gmail.com

This article has been accepted for publication and undergone full peer review but has not been through the copyediting, typesetting, pagination and proofreading process, which may lead to differences between this version and the Version of Record. Please cite this article as doi: 10.1002/MP.13861

This article is protected by copyright. All rights reserved 


\section{Abstract}

Purpose: To introduce a new algorithm -MicroCalc- for dose calculation by modeling microdosimetric energy depositions and the spectral fluence at each point of a particle beam. Proton beams are considered as a particular case of the general methodology. By comparing the results obtained against Monte Carlo computations we aim to validate the microdosimetric formalism presented here and in previous works.

Methods and material: In previous works, we developed a function on the energy for the average energy imparted to a microdosimetric site per event and a model to compute the energetic spectrum at each point of the patient image. The number of events in a voxel is estimated assuming a model in which the voxel is completely filled by microdosimetric sites. Then, dose at every voxel is computed by integrating the average energy imparted per event multiplied by the number of events per energy beam of the spectral distribution within the voxel. Our method is compared with the Proton Convolution Superposition (PCS) algorithm implemented in Eclipse $^{\mathrm{TM}}$ and the fast Monte Carlo code MCsquare, which is here considered the benchmark, for in-water calculations, using in both cases clinically validated beam data. Two clinical cases are considered: a brain and a prostate case.

Results: For a SOBP beam in water, the mean difference at the central axis found for MicroCalc is of $0.86 \%$ against $1.03 \%$ for PCS. Three-dimensional gamma analyses in the PTVs compared with MCsquare for criterion (3\%, $3 \mathrm{~mm}$ ) provide gamma index of $95.07 \%$ with MicroCalc versus $94.50 \%$ with PCS for the brain case and $99.90 \%$ versus $100.00 \%$, respectively, for the prostate case. For selected organs at risk in each case (brainstem and rectum), mean and maximum difference with respect to MCsquare dose are analyzed. In the brainstem, mean differences are 0.25 Gy (MicroCalc) vs 0.56 Gy (PCS), while for the rectum these values are $0.05 \mathrm{~Gy}$ (MicroCalc) vs $0.07 \mathrm{~Gy}$ (PCS).

Conclusions: The accuracy of MicroCalc seems to be, at least, not inferior to PCS, showing similar or better agreement with MCsquare in the considered cases. Additionally, the algorithm enables simultaneous computation of other quantities of interest. These results seem to validate the microdosimetric methodology in which the algorithm is based on.

Keywords: microdosimetry, dose calculation, proton therapy, dose algorithms 


\section{Introduction}

Microdosimetry is the study of the energy deposited by charged particles in microscopic structures. This framework was originally developed to evaluate the effects of radiation on cells and sub-structures of cells $^{1-3}$. Due to the stochastic nature of the interaction between charged particles, microdosimetry theory deals with distributions, rather than deterministic values, of energy imparted to such structures ${ }^{4}$. Lineal energy $(y)$ is a microdosimetric quantity, defined as the quotient between the energy imparted to a certain structure, called site, and the mean chord length of the particle in that site ${ }^{5}$. The average of the weighted distribution of this quantity is related to the macroscopic concept of Linear Energy Transfer (LET) ${ }^{6}$. Finally, LET is considered as one of the factors impacting the relative biological effectiveness (RBE) for each kind of radiation ${ }^{7-9}$.

On the other hand, specific energy $(z)$ is a different stochastic quantity, defined as the energy imparted to the site divided by the site mass. The average of the microdosimetric distribution of $z$, coincides with the macroscopic concept of absorbed dose ${ }^{10}$. Traditionally, distributions of specific energies for different types of radiation have been applied, for instance, to obtain radiobiological parameters defining the effect of a given test radiation ${ }^{11}$ or to characterize detectors responses ${ }^{12}$. However, it has never been used to calculate three-dimensional dose distributions in a treatment planning system (TPS) for ion therapy. We present a general methodology to analytically calculate dose based on specific energy, particularized to the proton radiotherapy case.

\section{Methods and Materials}

Starting from previous works ${ }^{13,14}$, we have created an analytical model for the dependency of the frequency average of the single-event distribution of specific energy $\bar{z}_{F}$ on the proton kinetic energy, i.e., a function $\bar{z}_{F}=\bar{Z}_{F}(E)$. The subscript $F$ indicates frequency-average, i.e., the average of the frequencies of appearances for each possible specific energy value in a single event. This subscript is traditionally included in contraposition to the dose-weighted average, $\bar{z}_{D}$, which results from weight each specific energy value for a single event according to the dose imparted during that event. $\bar{z}_{F}(E)$ needs to be integrated over the number of events occurred for each proton energy at each 
point of the calculation grid. Therefore, a dose distribution can be calculated as long as the information of the number of events per energy is known.

\section{Definitions in microdosimetry theory}

Given a beam of charged particles passing through matter, an event in microdosimetry is the process in which each independent particle track deposits energy by interacting with the atoms of the matter ${ }^{15}$. The event, by definition, includes the depositions of energy from the secondary particles (mainly electrons) generated along the particle track. A site is a sub-region of the matter traversed by the radiation beam in which energy deposits are scored. Sites can take different shapes and sizes and allows for characterizing the way in which energy is deposited spatially. The sum of all energy deposits in a site per each independent particle track is called single-event energy imparted, $\varepsilon_{s}$, and it is a stochastic quantity, whose actual value for each event depends on several factors ${ }^{16}$. Therefore, after a number of events, it is possible to obtain the single-event distribution of energy imparted, in terms of its probability density function, $f\left(\varepsilon_{s}\right)$, by counting the relative frequency of events with each value of energy imparted.

The quotient between the energy imparted $\varepsilon$ after a number of events and the mass $m$ of the site is called specific energy, $z=\varepsilon / m$. Then, the probability density function of the single-event distribution of specific energy, $f_{1}(z)$, can be obtained from $f\left(\varepsilon_{s}\right)$. The average of the single-event distribution of specific energy is noted by $\bar{z}_{F}$, where $F$ stands for frequency. For a given number of events, the specific energy for a site is obtained as the sum of the specific energies at each event. Therefore, as $z$ is also a stochastic quantity, if we consider different series of events, with average $v$, the distribution of the total specific energy can be expressed in terms of its probability density function, $f(z ; v)$. The dose absorbed by the site is not a stochastic quantity and coincides with the average of $f(z ; v)$, i.e.

$$
D=\int d z f(z ; v)=\bar{z}
$$

It is more interesting to consider the distribution of specific energy for a given dose $D, f(z ; D)$, rather than in terms of the average number of events $v$. This distribution gives the probability of obtaining a specific energy $z$ after a series of events when the absorbed dose is $D$. This distribution can be obtained from the single-event distribution of specific 
energy, $f_{1}(z)$, since the interactions between particles and matter can be described by a compound Poisson process ${ }^{10}$. Hence, the absorbed dose can be expressed in terms of the average of the single-event distribution of specific energy as

$$
D=v \bar{z}_{F}
$$

Accordingly, if the number of events occurring in a site and the mean specific energy per event are known, it is possible to calculate the corresponding absorbed dose.

\section{Spectral decomposition}

Equation (2) is valid for any beam regardless its particles or spectrum. However, it is challenging to analytically model $\bar{Z}_{F}$ for such a broad range of possibilities. A good approach consists of considering the single-event distributions of specific energy for monoenergetic beams of energy $E$, with average $\bar{z}_{F}=\bar{z}_{F}(E)$. Then, if the number of events $v(E)$ from particles with energy $E$ is known, the absorbed dose can be calculated as

$$
D=\int v(E) \bar{z}_{F}(E) \mathrm{d} E
$$

Model for $\bar{Z}_{F}(E)$

As said above, the single-event specific energy is related to $\bar{\varepsilon}_{s}$. Equivalently, the average of its single-event distribution is given by

$$
\bar{z}_{F}(E)=\frac{\bar{\varepsilon}_{S}(E)}{m} .
$$

The average energy imparted to a site at each particle energy depends on three factors: (a) the stopping power of the particle, (b) how long is the particle track within the site -segment length-, and (c) the probability that secondary electrons generated by the particle leave the site. The trial function for $\bar{\varepsilon}_{S}(E)$ should be, firstly, proportional to the stopping power, which in turn depends on the particle energy. It also should contain a term to consider the losses of energy imparted when the range of secondary electrons is larger than the site dimensions. And finally, it needs to decrease as the particle track becomes shorter. In previous works we have explored how to use Monte Carlo simulations to produce microdosimetric data ${ }^{13}$ and what functions can be used to characterize microdosimetric quantities ${ }^{14}$. A function meeting all the referred requirements is: 


$$
\bar{\varepsilon}_{S}(E)=C \cdot \operatorname{erf}\left(k E^{q}\right) \cdot \log \left(h_{P, m} E+b\right) \cdot \frac{\alpha_{P, m} \cdot E^{\left(p_{P, m}-1\right)}+e}{\alpha_{P, m} \cdot E^{p_{P, m}}+2 D_{s} / 3}
$$

where $C, b$ and $e$ are free parameters of the function whereas $h_{P, m}, \alpha_{P, m}$ and $p_{P, m}$ depend on the considered particle and medium and $D_{s}$ depends on the site dimension.

The mass of the site can be obtained from its volume and the density of the considered medium. Since dose to water is the quantity normally used in a TPS for dose calculations, the site is considered to be made of liquid water.

Number of events per energy, $v(E)$ : a model for the voxel-site relation

By definition, the distribution of average number of events per energy $v(E)$ should coincide with the spectral distribution at the site, i.e., the number of particles of each energy impinging on the site. However, we are normally interested in calculating dose in a voxelized geometry, being the voxel considerably larger than the typical microscopic sites. Therefore, we need to firstly consider the number of events occurring in the voxel. For this, we assume the voxel is represented by an arrangement of sites in a rectangular grid, as Figure 1 shows for the case of spherical sites. Let $N_{t}$ be the number of sites in the voxel contained on a layer perpendicular to the particle track, where $t$ stands for transversal. Then, if the transversal cross section of a site is $\sigma_{s}$, the total cross section in which events are produced is $N_{t} \sigma_{s}$ so that the number of events in the voxel is proportional to $\Phi_{E}(E) N_{t} \sigma_{s}$, where $\Phi_{E}(E)$ is the spectral fluence of particles. Note that this is valid as long as we can assume that the number of indirect events (i.e. produced just by secondary particles) is negligible with respect to the number of direct events (i.e. produced by primary protons), which is expected to be the case for any voxel with dose relevant in radiotherapy.

An additional aspect needs to be taken into consideration. Let $d_{V}$ be the dimension of the voxel parallel to the direction of the particle tracks and $d_{s}$ the maximum dimension of the site in the same direction. Then, the number of sites aligned within the voxel will be $N_{l}=d_{V} / d_{s}$, where $l$ stands for longitudinal. If the incidental particle energy is such that its range is longer than $d_{V}$, then it will exit the voxel producing an event in each site on its way. Therefore, the number of events will be $v=N_{l}$. However, if the range of the particle is shorter than $d_{V}$, the number of events produced by that particle is fewer since the proton stops before reaching the final sites. We assume, in that case, that the number of events 
produced by that particle is proportional to its CSDA range, $R(E)$, so the number of events in the voxel per energy, $v_{V}(E)$, is:

$$
v_{V}(E)=\Phi_{E}(E) N_{t} \sigma_{s} \max \left(\frac{d_{V}}{d_{s}},\left\lceil\frac{R(E)}{d_{s}}\right\rceil\right),
$$

where $\lceil x\rceil$ means the least integer greater than or equal to $x$, since the number of events is an integer quantity. However, according to equation (2), we are interested in the average number of events per site rather than in the complete voxel. As the total number of sites in the voxel is $N_{t} N_{l}$, the average number of events per site is given by

$$
v(E)=\frac{v_{V}(E)}{N_{t} N_{l}}=\frac{1}{N_{l}} \Phi_{E}(E) \sigma_{s} \max \left(\frac{d_{V}}{d_{s}},\left\lceil\frac{R(E)}{d_{s}}\right\rceil\right) .
$$

A particular case: protons in spherical sites

All shown so far remains as valid regardless the particle species, and the site's shape or dimension considered. Now, we introduce a particular case to illustrate the performance of the method. The analysis beyond this point is focused on proton clinical beams and spherical sites with diameter $d$. For this specific case, the longitudinal length of the site equals to the site diameter, $d_{s}=d$, and so it does the site-dependent parameter on the expression for $\bar{\varepsilon}_{s}$ in equation (5): $D_{s}=d .^{14}$ In order to obtain the rest of parameters in equation (5), we performed simulations with Geant4-DNA ${ }^{17-20}$ of mono-energetic protons in spherical sites made of liquid water up to $100 \mathrm{MeV}$, which is the upper limit the code has for proton transport. To assess the influence of the site dimension in the calculation, we have considered three different cases for the site diameter: $1 \mu \mathrm{m}, 5 \mu \mathrm{m}$ and $10 \mu \mathrm{m}$. To calculate $\bar{\varepsilon}_{S}(E)$, for proton energies higher than $100 \mathrm{MeV}$ we assume the validity of equation (5) can be extrapolated to those energies, as our trial function has a smooth behavior at such energies.

Kernel for the spectral fluence of proton beams

Once $\bar{\varepsilon}_{S}$, and therefore $\bar{z}_{F}$, are obtained, the only quantity remaining to be determined is the spectral fluence density, $\Phi_{E}(E)$. Again, this means the number of protons of each energy per unit area traversing each voxel in which the CT image of a patient is divided. This information can be obtained through Monte Carlo simulations of the proton 
transport in the patient. Some TPS are able to provide that information ${ }^{21,22}$. However, we have developed a kernel-based algorithm for the spectral fluence at each point given a proton arriving to the patient surface. Here we provide an outline of this, although it is fully presented in a separate work ${ }^{23}$. Different simulations of the transport of protons in water were carried out by using an application based on the Monte Carlo toolkit Geant4 ${ }^{24-26}$. Several values for the energy have been selected in the range of clinical use in proton radiotherapy: from $50 \mathrm{MeV}$ up to $250 \mathrm{MeV}$.

A distinction between primary and secondary protons is made to develop independent models for their transport in water. Primary protons are those which only undergo elastic collisions with the atoms of matter meanwhile secondary protons are those coming from inelastic collisions with nuclei. For each one, the model is composed by three elements: (i) given an initial fluence of protons $\Phi_{0}$ penetrating in water, the fraction of primary $\Phi_{P P}(d) / \Phi_{0}$ and secondary protons $\Phi_{S P}(d) / \Phi_{0}$ in a whole transversal plane at a given depth $d$; (ii) the lateral distribution of primary $\mathrm{d} \phi_{P P}(r ; d) / \mathrm{d} r$ and secondary protons $\mathrm{d} \phi_{S P}(r ; d) / \mathrm{d} r$ at each depth and each distance $r$ to the central axis of the beam; and (iii) the energy spectrum for primary $\mathrm{d} \phi_{P P}(E ; r, d) / \mathrm{d} E$ and secondary $\mathrm{d} \phi_{S P}(E ; r, d) / \mathrm{d} E$ at each lateral position and each depth. For the first case, we use the function proposed in the work of Ulmer ${ }^{27}$ for the primary protons, whereas the proportion of secondary protons at each depth $\Phi_{S P}(d)$ is modeled by a phenomenological function ${ }^{23}$ :

$$
\frac{\Phi_{S P}(d)}{\Phi_{0}}=\left(a d-b d^{c}\right) \cdot \frac{1}{2}\left(1+\operatorname{erf}\left(\frac{R(E)-d}{\sqrt{2} \tau}\right)\right)
$$

where $a, b$ and $c$ are parameters to be fitted dependent on the beam energy, $R(E)$ is the CSDA range corresponding to the initial beam energy and $\tau$ is the same parameter than used by Ulmer ${ }^{27}$, related to the range straggling of the beam.

For the lateral distribution, we assume that a single Gaussian centered at the beam axis is enough to model the spread in the fluence of primary protons, but not for the secondary ones. Hence, a Double Gaussian is proposed as a better function to model the latter case. Finally, for the sake of simplicity, the energy spectrum for both primaries and secondaries is modeled as a single Gaussian whose mean energy is given by the residual 
range (i.e., $R(E)-d$ ). Then, the kernel of spectral distribution at a voxel with entrance surface $\mathrm{d} S$ at the position $(d, r)$ is given by

$$
\Phi_{E}^{\text {Kernel }}(E ; d, r) \mathrm{d} S=\sum_{i=P P, S P} \frac{\Phi_{i}(d)}{\Phi_{0}} \frac{\mathrm{d} \phi_{i}(r ; d)}{\mathrm{d} r} \frac{\mathrm{d} \phi_{i}(E ; r, d)}{\mathrm{d} E} \mathrm{~d} S
$$

where $i$ refers to protons and secondary protons. Note that both $\mathrm{d} \phi_{i}(r ; d) / \mathrm{d} r$ and $\mathrm{d} \phi_{i}(E ; r, z) / \mathrm{d} E$ are normalized to the unit so that the argument in the summation provides the probability for a proton to have an energy $E$ at the position $(d, r)$. Finally, to obtain the spectral fluence density for a broad beam it is necessary to convolve two-dimensionally the spectral fluence density of the broad beam coming onto the patient surface, $\Phi_{E}^{\text {Surface }}(E ; r)$, with the kernel presented in equation (9):

$$
\Phi_{\mathrm{E}}(E ; d, r)=\int \Phi_{E}^{\text {Surface }}\left(E ; r^{\prime}\right) \Phi_{E}^{\text {Kernel }}\left(E ; d, r-r^{\prime}\right) \mathrm{d} r^{\prime}
$$

This model for the proton transport only considers liquid water. Transport through other media is here considered by replacing in equation (9) the depth in water, $d$, by the corresponding water-equivalent thickness $d_{W E T}$ resulting from the beam raytracing.

\section{Dose distributions comparisons}

All above have been implemented in a script for the Eclipse ${ }^{\mathrm{TM}}$ TPS V15.6 (Varian Medical Systems, Palo Alto, CA, USA) for Proton Beam Scanning (PBS) treatments, called hereinafter 'MicroCalc' or ' $\mu$ Calc'. This allows us for producing actual dose calculations in three-dimensional geometries. The TPS provides the information about the beam lateral width -to be convolved with our lateral model-. On the other hand, the number of protons prior to arrive to the patient surface can be estimated according to the number of monitor units (MU) for each $\operatorname{spot}^{21}$ so that we can obtain $\Phi_{E}^{\text {Surface }}(E ; r)$. We present comparisons of dose distributions obtained by: (a) the microdosimetric method presented in equations (3-7) together with the kernel for the proton fluence summarized in equation (9); (b) the analytical Proton Convolution-Superposition (PCS) algorithm ${ }^{28}$ provided by Eclipse $^{\mathrm{TM}}$; and (c) Monte Carlo simulations with the open source fast MC code MCsquare ${ }^{29}$, which is used as a benchmark in this work as it has been validated elsewhere ${ }^{30,31}$. 
We have considered three different situations with different complexities: (1) single energy beams broad enough to provide lateral equilibrium at the central axis, computed in water; (2) a single beam conforming a spread-out Bragg peak (SOBP) with energy layers from $118 \mathrm{MeV}$ to $175 \mathrm{MeV}$ in a water box; and (3) clinical plans for a brain cancer case with three beams and a prostate case with two lateral beams. In the last cases, global 3D gamma analyses in the PTV structures with interpolation are performed to evaluate the differences between analytical methods (MicroCalc and PCS) and the MC calculation (MCsquare) with the MATLAB-based environment OpenREGGUI. The criterion for those gamma analyses is $(3 \%, 3 \mathrm{~mm})$ considering the MCsquare distribution as the reference in all cases. To evaluate the accuracy of the dose calculations in organs at risk, we select an adjacent structure to the PTV in each case to compute differences with respect to MCsquare: brainstem in the brain case and rectum in the prostate case.

\section{Results}

Three different beams are considered to show their dose profiles at central axis in Fig. 2: single energy layers of $110 \mathrm{MeV}, 160 \mathrm{MeV}$ and $210 \mathrm{MeV}$.

Fig. 3 shows the dose profiles at the central axis for a beam with energy layers from $118 \mathrm{MeV}$ to $175 \mathrm{MeV}$ optimized in Eclipse ${ }^{\mathrm{TM}}$ to produce a uniform dose distribution in a target of $10 \mathrm{~cm}$ in length and whose distal edge is placed at $20 \mathrm{~cm}$ in depth. The mean absolute difference along the profile between MCsquare and MicroCalc is $0.86 \%$ while the mean difference between MCsquare and PCS algorithm is $1.03 \%$.

A clinical case for brain cancer is presented in Fig. 4. Dose distributions for MCsquare, MicroCalc and PCS on an axial plane located at the middle of the PTV are shown in Fig. 4 (a), (b) and (c), respectively. Differences between MCsquare and (i) MicroCalc and (ii) PCS can be seen in Fig. 4 (d) and (e), respectively. Three-dimensional gamma analysis with criterion ( $3 \mathrm{~mm}, 3 \%$ ) in the PTV between MicroCalc and MCsquare shows a gamma index of $95.07 \%$ while the gamma analysis for differences between PCS and MCsquare is 94.50\%. The same procedure in the CTV produces results of $95.10 \%$ and $95.03 \%$, respectively. Mean differences in the dose distribution for the brainstem structure are found to be 0.25 Gy between MicroCalc and MCsquare and 0.56 Gy between PCS and MCsquare, respectively.

This article is protected by copyright. All rights reserved 
A similar analysis is shown in Figure 5 for a prostate case. Results for dose distributions for an axial plane, located at the middle of the PTV are shown for MCsquare, MicroCalc and PCS calculations, respectively. Again, 3D gamma analysis in the PTV contour with criterion $(3 \%, 3 \mathrm{~mm})$ is performed by comparing MCsquare to MicroCalc and PCS, respectively. The gamma index is $99.90 \%$ for the comparison MCsquare-MicroCalc and $100 \%$ for the comparison MCsquare-PCS. In the rectum, the mean differences in dose between MicroCalc or PCS and MCsquare are 0.05 Gy and $0.07 \mathrm{~Gy}$.

\section{Discussion}

Whereas the number of results presented here is not large enough to assess the significance of the goodness for MicroCalc dose calculation, the performance of the proposed model is found comparable to the differences found between MCsquare and the clinically validated algorithm PCS. The ability to reproduce dose ratio between Bragg peak and plateau zone, as well as the beam range, is clearly observable in Fig. 2 . When not considering only pristine Bragg peaks, a better agreement with MCsquare is found for MicroCalc at the SOBP than PCS. This better agreement shows up both in the mean difference along the profile at the central axis and the shape of the curve itself, slightly nonuniform as opposed to the PCS algorithm shows. Results shown in Fig. 3 are reproduced in both clinical cases presented (Fig. 4 and Fig. 5). Comparable (3\%, 3mm) gamma index are found for the 3D analysis inside the PTV. Also, fewer hot spots are produced beyond that structure for MicroCalc than for PCS, as observable by comparing Figs. 4 and 5 (d) and (e), even though both analytical algorithms tend to underestimate the dose beyond distal edges. On the other hand, an underestimation of the dose is observed at the penumbra of the beams for both analytical algorithms (Fig. 5 (d) and (e)). However, the beam model employed in Eclipse ${ }^{\mathrm{TM}}$ to analytically compute dose uses a single Gaussian to characterize the lateral profile whereas it has been pointed out that might be insufficient to reproduce adequately transversal dose distributions ${ }^{32}$.

As pointed out, the function in equation (5) that characterize the energy deposition on the proton energy is extrapolated beyond $100 \mathrm{MeV}^{14}$ because of the limitation existing in the current version of Geant4-DNA. That lack of actual data for higher energies than this limit is a potential explanation for the observed differences at the entrance with MCsquare 
in Fig. 3. Besides that, in our current calculations, only protons and alpha particles are considered thus dose coming from heavy particles produced in heavy nuclear interaction is not considered. According to Grassberger and Paganetti ${ }^{33}$, the contribution of those is relatively higher at the entrance so that this effect may also participate on the found differences at that area. An important advantage of MicroCalc is that it allows for incorporating the contributions of different particles independently. This can be done by characterizing the microdosimetric energy depositions in a similar way as for protons.

Even in complex non-homogeneous cases as shown in Fig. 4, the dose calculation provided by MicroCalc is able to produce results close to those provided by MCsquare. However, larger inaccuracies may appear when considering greater tissue variabilities along the path beam, as seen at the left posterior cavity in Fig. 4(d). Nonetheless, the approach employed to take into consideration lack of homogeneity is relatively simple, i.e., only a displacement of the calculated fluence according to the water-equivalent depth. Therefore, a potential improvement of this work consists of implementing a more sophisticated method to overcome large inhomogeneities situations.

Besides the accuracy of the calculation, at least comparable to other analytical methods, two main reasons make MicroCalc a powerful tool compared to pencil beam algorithms: (i) it can calculate several quantities simultaneously ${ }^{23}$; and (ii) it is modular, i.e. different particles can be added independently. Both reasons are based on the idea of transporting protons in water while keeping their frequency and spectrum information, which makes MicroCalc closer to actual MC methods. As shown in previous works ${ }^{14}$, not only dose but also three-dimensional distributions of microdosimetric quantities might be obtained in combination with modeled energy-dependent functions in a similar way than shown here. Furthermore, other macroscopic quantities as dose-averaged LET can be also calculated $^{23}$ based on stopping power curves, and even combinations of all those functions for optimization purposes (e.g. dose-LET product) may be computed in a straightforward way.

\section{Conclusions}

A new analytical algorithm -MicroCalc- to calculate dose is explicitly introduced and detailed in this work. The accuracy of this method is, at least, not inferior to the Proton 
Convolution Superposition (PCS) algorithm, already clinically validated in Eclipse ${ }^{\mathrm{TM}}$. This seems to corroborate the methodology employed in this work as well as, partially, the microdosimetric functions developed in our previous work ${ }^{14}$. On top of that, MicroCalc provides the possibility of separating different particles contributions and simultaneously computing several quantities of interest, which conceptually makes it similar to Monte Carlo techniques.

\section{Acknowledgements}

This project is supported by Varian Medical Systems, Palo Alto, California; M. A. Cortés-Giraldo has been funded by the Spanish Ministry of Economy and Competitiveness under Grant No. FPA2016-77689-C2-1-R, and has no relation with any private funding. Marie Cohilis is supported by the Télévie Grant from the Belgian "Fonds National pour la Recherche Scientifique" F.R.S-FNRS (Grant No. 7450517F). 


\section{References}

1. Rossi HH. Spatial Distribution of Energy Deposition by lonizing Radiation Source. Radiat Res. 1960;2(Suplement):290-299.

2. Rossi HH. Microdosimetry. Biophys Asp Radiat Qual IAEA, Tech Reports Ser. 1966;58.

3. Kellerer AM, Rossi HH. The Theory of Dual Radiation Action. In: Ebert M, Howard A, eds. Current Topics in Radiation Research. Volume VIII. Manchester, U.K.: American Elsevier Publishing Company; 1974:85-156.

4. Kellerer AM, Chmelevsky D. Concepts of microdosimetry - II. Probability distributions of the microdosimetry variables. Radiat Environ Biophys. 1975;12(3):205-216. doi:10.1007/BF01327348

5. Kellerer AM, Chmelevsky D. Concepts of microdosimetry - I. Quantities. Radiat Environ Biophys. 1975;12(2):61-69.

6. Kellerer AM, Chmelevsky D. Concepts of microdosimetry - III. Mean values of the microdosimetric distributions. Radiat Environ Biophys. 1975;12(4):321-335. doi:10.1007/BF01323420

7. Belli M, Cera F, Cherubini R, et al. RBE-LET relationships for cell inactivation and mutation induced by low energy protons in V79 cells: Further results at the LNL facility. Int J Radiat Biol. 1998;74(4):501-509. doi:10.1080/095530098141375

8. Hawkins RB. A Microdosimetric-Kinetic Model for the Effect of Non-Poisson Distribution of Lethal Lesions on the Variation of RBE with LET. Radiat Res. 2003;160(1):61-69. doi:10.1667/RR3010

9. Barendsen GW. The Relationships between RBE and LET for Different Types of Lethal Damage in Mammalian Cells: Biophysical and Molecular Mechanisms. Radiat Res. 1994;139(3):257-270. doi:10.2307/3578823

10. Kellerer AM. Fundamentals of microdosimetry. In: Kase KR, Bjarngard BE, Attix FH, eds. The Dosimetry of Ionization Radiation. Volume I. Academic Press, Inc.; 1985:77162.

11. Rossi HH, Zaider M. Microdosimetry and Its Applications. Springer; 1996.

This article is protected by copyright. All rights reserved 
12. Olko P. The microdosimetric one-hit detector model for calculating the response of solid state detectors. Radiat Meas. 2002;35(3):255-267. doi:10.1016/S13504487(01)00292-X

13. Bertolet A, Baratto-Roldán A, Barbieri S, Baiocco G, Carabe A, Cortés-Giraldo MA. Dose-averaged LET calculation for proton track segments using microdosimetric Monte Carlo simulations. Med Phys. 2019;46(9):4184-4192. doi:10.1002/mp.13643

14. Bertolet A, Baratto-Roldán A, Cortés-Giraldo MA, Carabe-Fernandez A. Segmentaveraged LET concept and analytical calculation from microdosimetric quantities in proton radiation therapy. Med Phys. 2019;46(9):4204-4214. doi:10.1002/mp.13673

15. ICRU. Report 36. Microdosimetry.; 1983.

16. Kellerer AM. Analysis of Patterns of Energy Deposition. In: Ebert HG, ed. Second Symposium on Microdosimetry. Stresa (Italy); 1970:107-136.

17. Incerti S, Baldacchino G, Bernal M, et al. The Geant4-DNA project. Int J Model Simulation, Sci Comput. 2010;1(2):157. doi:10.1142/S1793962310000122

18. Incerti S, Ivanchenko A, Karamitros M, et al. Comparison of GEANT4 very low energy cross section models with experimental data in water. Med Phys. 2010;37(9):46924708. doi:10.1118/1.3476457

19. Bernal MAA, Bordage MCC, Brown JMCMC, et al. Track structure modeling in liquid water: A review of the Geant4-DNA very low energy extension of the Geant4 Monte Carlo simulation toolkit. Phys Medica. 2015;31(8):861-874.

doi:10.1016/j.ejmp.2015.10.087

20. Incerti S, Kyriakou I, Bernal MA, et al. Geant4-DNA example applications for track structure simulations in liquid water: A report from the Geant4-DNA Project. Med Phys. 2018;45(8):e722-e739. doi:10.1002/mp.13048

21. Lin L, Huang S, Kang $M$, et al. A benchmarking method to evaluate the accuracy of a commercial proton monte carlo pencil beam scanning treatment planning system. $J$ Appl Clin Med Phys. 2017;18(2):44-49. doi:10.1002/acm2.12043

22. Saini J, Maes D, Egan A, et al. Dosimetric evaluation of a commercial proton spot 
scanning Monte-Carlo dose algorithm: Comparisons against measurements and simulations. Phys Med Biol. 2017;62(19):7659-7681. doi:10.1088/1361-6560/aa82a5

23. Bertolet A, Cortés-Giraldo MA, Souris K, Carabe-Fernandez A. A kernel-based algorithm for fluence and spectral fluence in clinical proton beams to calculate doseaveraged LET and other dosimetric quantities of interest. Med Phys [SUBMITTED]. 2019.

24. Agostinelli S, Allison J, Amako K, et al. Geant4-a simulation toolkit. Nucl Instruments Methods Phys Res Sect A Accel Spectrometers, Detect Assoc Equip. 2003;506(3):250303. doi:10.1016/S0168-9002(03)01368-8

25. Allison J, Amako K, Apostolakis J, et al. Geant4 developments and applications. IEEE Trans Nucl Sci. 2006;53(1):270-278. doi:10.1109/TNS.2006.869826

26. Allison J, Amako K, Apostolakis J, et al. Recent developments in Geant4. Nucl Instruments Methods Phys Res Sect A Accel Spectrometers, Detect Assoc Equip. 2016;835:186-225. doi:10.1016/J.NIMA.2016.06.125

27. Ulmer $\mathrm{W}$. Theoretical aspects of energy-range relations, stopping power and energy straggling of protons. Radiat Phys Chem. 2007;76(7):1089-1107. doi:10.1016/j.radphyschem.2007.02.083

28. Varian. Eclipse Proton Algorithms Reference Guide, v13.7. 2015.

29. Souris K, Lee JA, Sterpin E. Fast multipurpose Monte Carlo simulation for proton therapy using multi- and many-core CPU architectures. Med Phys. 2016;43(4):17001712. doi:10.1118/1.4943377

30. Sorriaux J, Testa M, Paganetti $H$, et al. Experimental assessment of proton dose calculation accuracy in inhomogeneous media. Phys Medica. 2017;38:10-15. doi:10.1016/j.ejmp.2017.04.020

31. Huang S, Kang M, Souris K, et al. Validation and clinical implementation of an accurate Monte Carlo code for pencil beam scanning proton therapy. J App/ Clin Med Phys. 2018;19(5):558-572. doi:10.1002/acm2.12420

32. Bellinzona VE, Ciocca M, Embriaco A, et al. On the parametrization of lateral dose 
profiles in proton radiation therapy. Phys Medica. 2015;31(5):484-492. doi:10.1016/j.ejmp.2015.05.004

33. Grassberger C, Paganetti H. Elevated LET components in clinical proton beams. Phys Med Biol. 2011;56(20):6677-6691. doi:10.1088/0031-9155/56/20/011 


\section{FIGURE CAPTIONS}

Figure 1. Model for the internal structure of a voxel composed by spherical sites. Protons characterized by a given fluence density impinge on the frontal face of the voxel and particles interact with the row of sites along their track.

Figure 2. Dose profiles at the central axis for single energy layers beams with energies 110 $\mathrm{MeV}, 160 \mathrm{MeV}$ and $210 \mathrm{MeV}$ calculated by employing the MicroCalc script, the PCS algorithm in Eclipse ${ }^{\mathrm{TM}}$ and the MC code MCsquare.

Figure 3. Dose profiles at central axis for a spread-out Bragg peak with maximum range at $20 \mathrm{~cm}$ in depth and modulation of $10 \mathrm{~cm}$ calculated by employing the MicroCalc script, the PCS algorithm in Eclipse ${ }^{\mathrm{TM}}$ and the MC code MCsquare.

Figure 4. Dose distributions on an axial plane for a brain case for (a) MCsquare, (b) MicroCalc and (c) PCS algorithm. The yellow contour depicts the PTV, with prescription dose of $54 \mathrm{~Gy}$. On the color map, blue color represents low dose (near $0 \mathrm{~Gy}$ ) while most intense red color represents 54 Gy. Differences between (d) MicroCalc and MCsquare and (e) PCS and MCsquare are also shown. In these color maps, most intense blue shows MCsquare dose is higher by $5 \mathrm{~Gy}$, most intense red means MCsquare dose is lower by 5 Gy and translucent means no difference.

Figure 5. Dose distributions on an axial plane at the middle of the PTV for a prostate case for (a) MCsquare, (b) MicroCalc and (c) PCS algorithm. The yellow contour depicts the PTV, with prescription dose of $68 \mathrm{~Gy}$. On the color map, blue color represents low dose (near $0 \mathrm{~Gy}$ ) while most intense red color represents $68 \mathrm{~Gy}$. Differences between (d) MicroCalc and MCsquare and (e) PCS and MCsquare are also shown. In these color maps, most intense blue shows MCsquare dose is lower by $3.5 \mathrm{~Gy}$, most intense red means MCsquare dose is higher by 3.5 Gy and white means no difference.

\section{LEGENDS}

Figure 2

$-\mu$ Calc.

- - PCS algorithm.

This article is protected by copyright. All rights reserved 
$\rightarrow$ MCsquare.

Figure 3

$-\mu$ Calc.

- - PCS algorithm.

MCsquare.

This article is protected by copyright. All rights reserved 


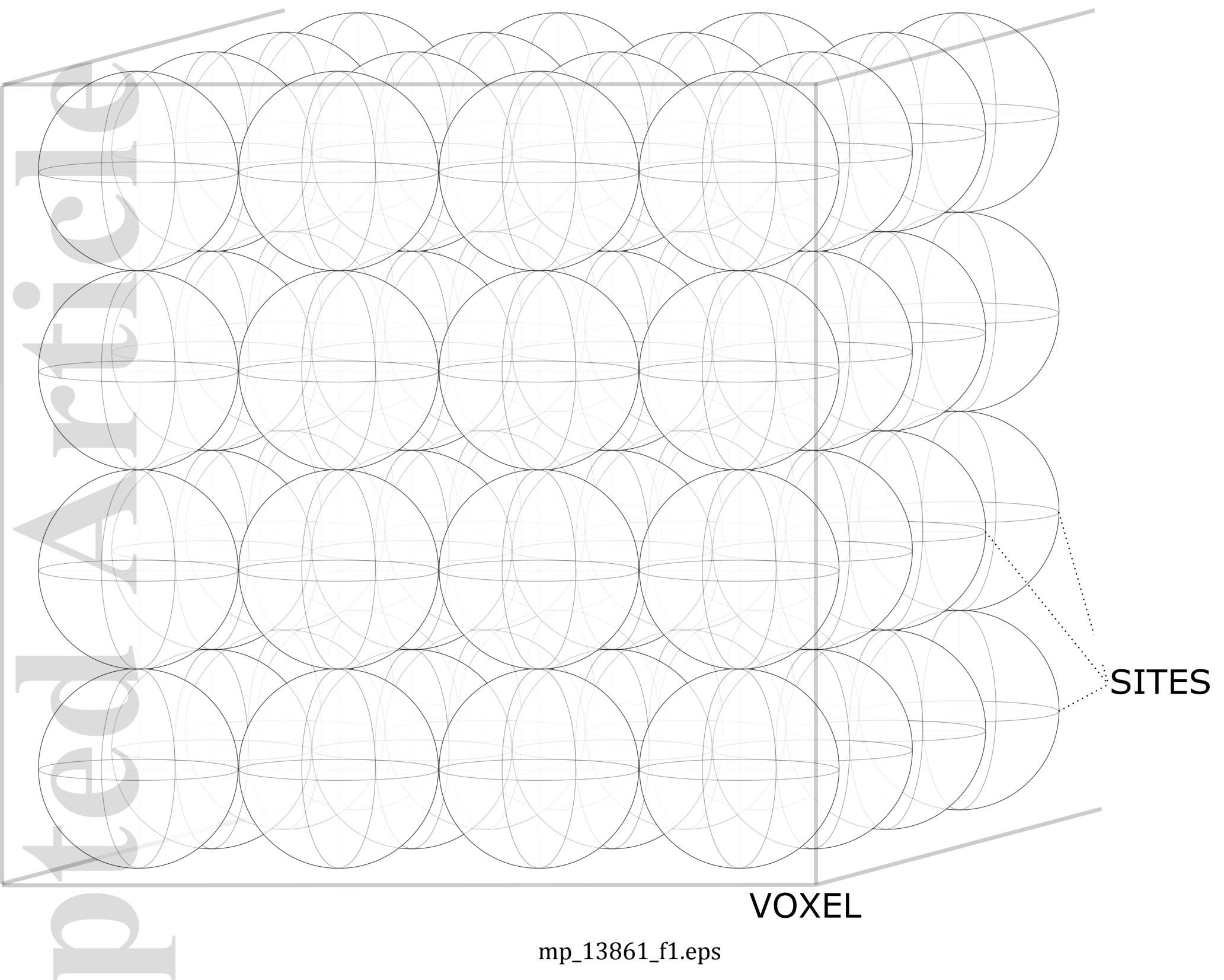

This article is protected by copyright. All rights reserved 


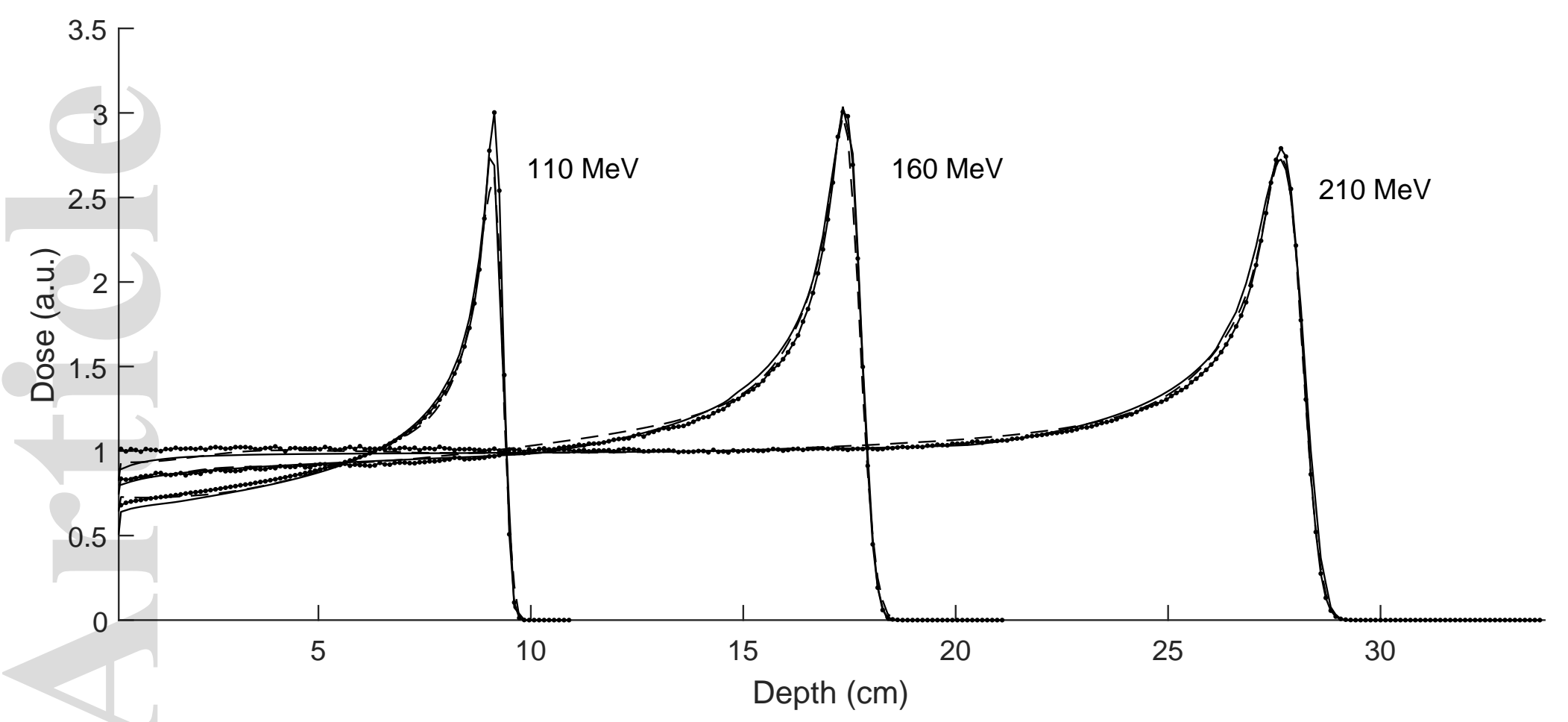

mp_13861_f2.eps 
(a)

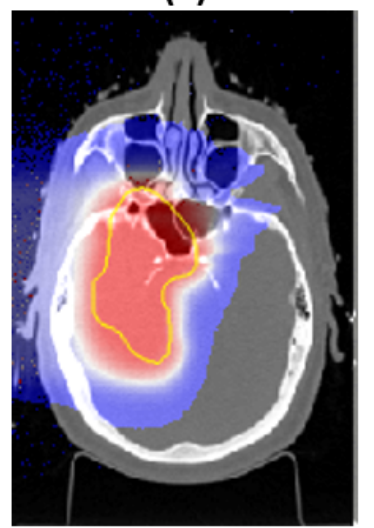

(d)

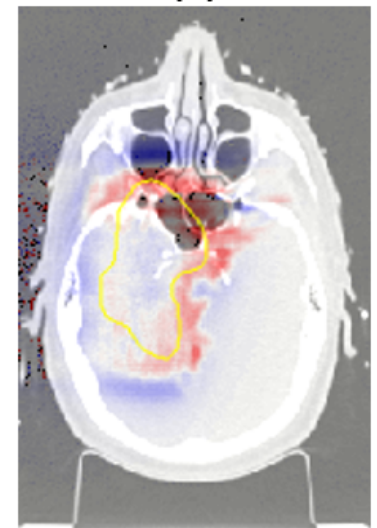

(b)

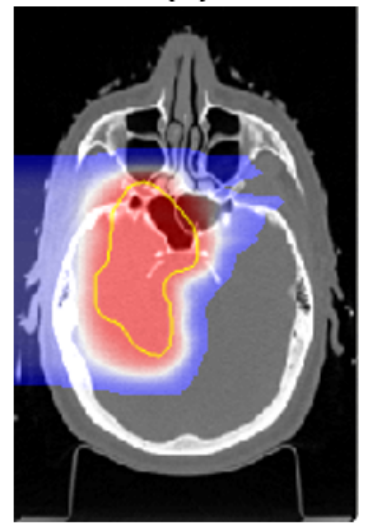

(e)

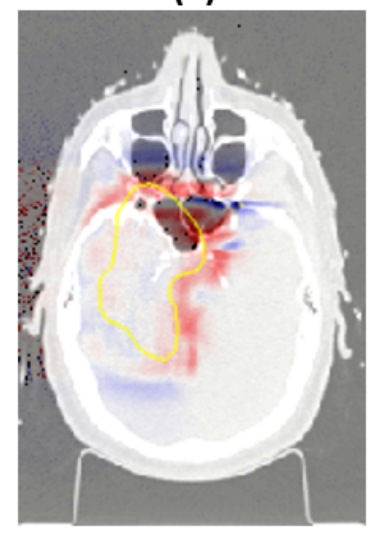

mp_13861_f4.tif
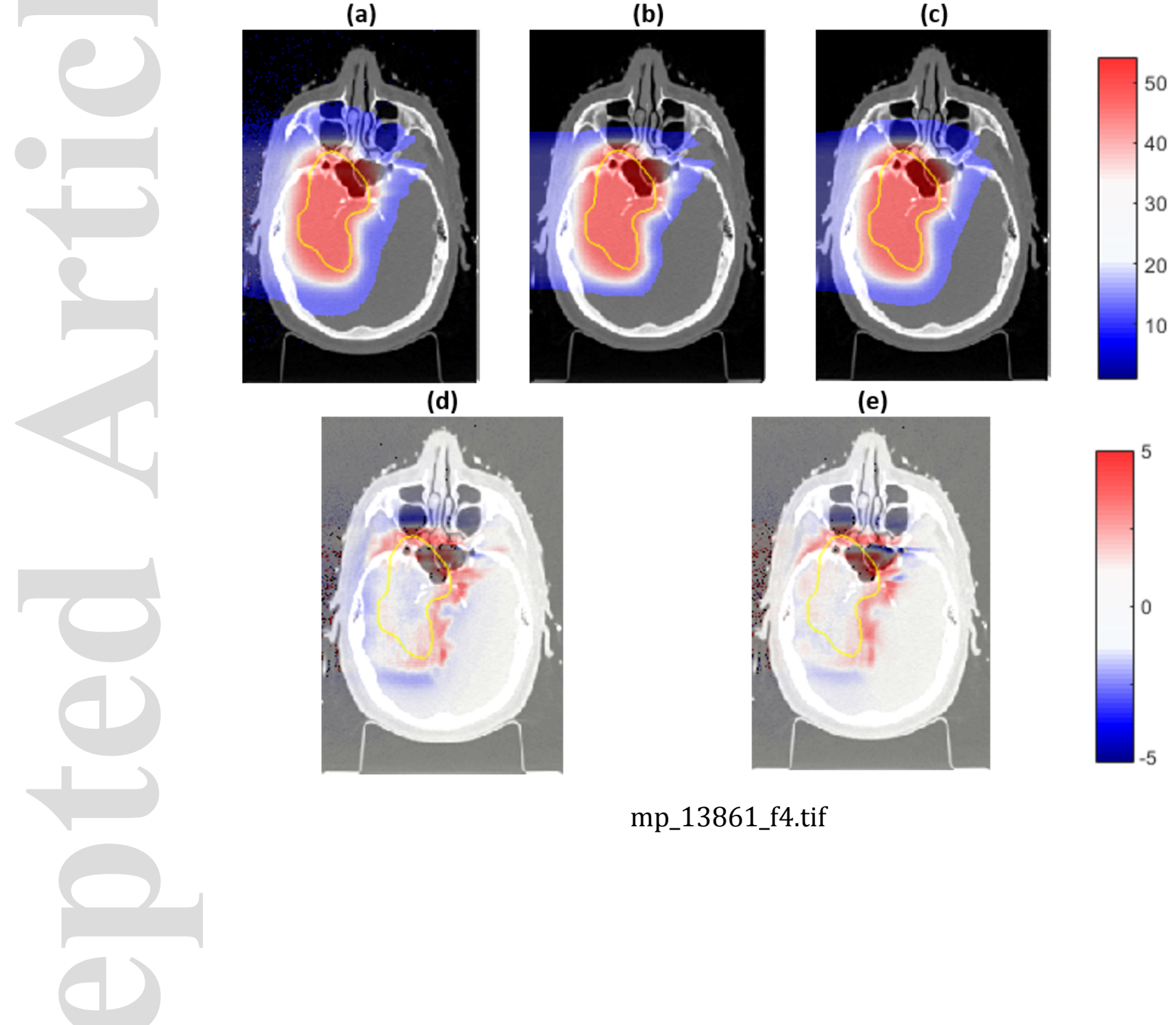

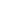
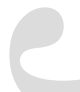

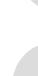
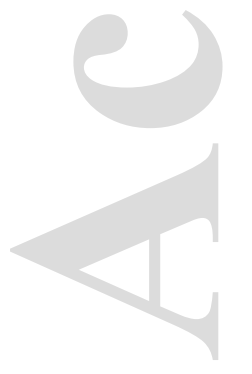

This article is protected by copyright. All rights reserved 
(a)

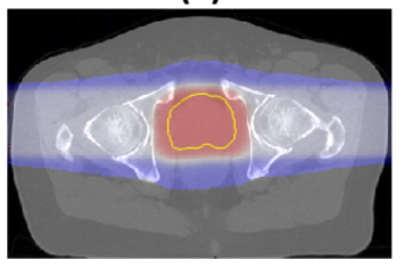

(d)

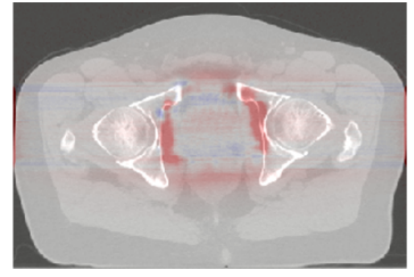

(b)
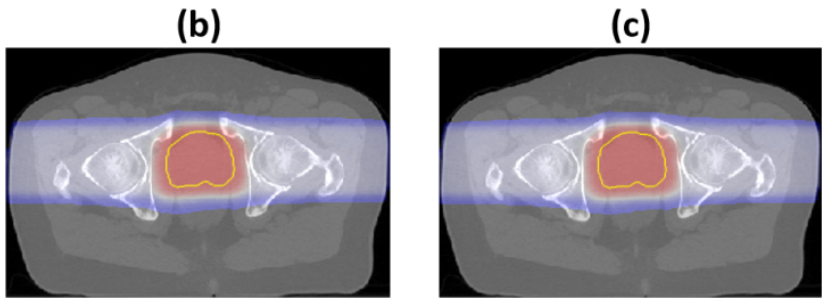

(e)

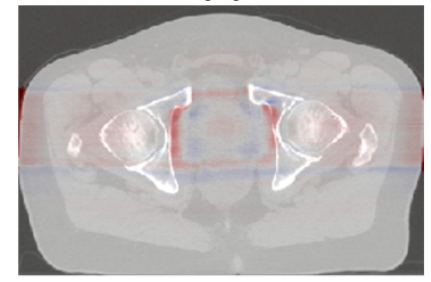

mp_13861_f5.tif 